\title{
Maternal microbiota Bifidobacterium promotes placental morphogenesis, nutrient transport and fetal growth in mice
}

Authors: Jorge Lopez-Tello ${ }^{1 *}$, Zoe Schofield ${ }^{2 *}$, Raymond Kiu², Matthew J. Dalby², Douwe van Sinderen ${ }^{3}$, Gwénaëlle Le Gall ${ }^{4}$, Amanda N Sferruzzi-Perri ${ }^{1 \dagger}$, Lindsay $\mathrm{J} \mathrm{Hall}^{2,5 \dagger}$

\section{Affiliations:}

${ }^{1}$ Department of Physiology, Development, and Neuroscience, Centre for Trophoblast Research, University of Cambridge, Cambridge, UK

${ }^{2}$ Gut Microbes \& Health, Quadram Institute Bioscience, Norwich Research Park, Norwich, UK ${ }^{3}$ APC Microbiome Institute, University College Cork, Cork, Ireland

${ }^{4}$ Norwich Medical School, University of East Anglia, Bob Champion Research and Education Building, James Watson Road, Norwich Research Park, Norwich NR4 7UQ, UK

${ }^{5}$ Chair of Intestinal Microbiome, School of Life Sciences, ZIEL - Institute for Food \& Health, Technical University of Munich, Freising, Germany

${ }^{*}$ Contributed equally

\section{Corresponding author names:}

Jorge Lopez-Tello (jl898@cam.ac.uk)

Amanda N. Sferruzzi-Perri (ans48@cam.ac.uk)

Lindsay J Hall (Lindsay.Hall@tum.de I Lindsay.Hall@quadram.ac.uk)

Author Contributions: JL-T, ZS, ANS-P, LJH designed research; JL-T, ZS, RK, GLG conducted research, JL-T, ZS, RK, MJD contributed analytic tools and performed analysis; DvS contributed reagents; JL-T, ZS, ANS-P, LJH wrote the paper with feedback from all the authors.

Competing Interest Statement: The authors declare that they have no competing interests.

Classification: Biological Sciences - Physiology

Keywords: Pregnancy, Placenta, Microbiota, Fetus, Bifidobacterium

\section{This file includes:}

Main Text: 5,454 (introduction, results, discussion and methods)

Main Figures: 3

Tables: 1

Supplementary Figures: 4 
Significance: Metabolism is highly influenced by the gut microbiota, which is particularly important during gestation, when key metabolites are used for feto-placental growth. However, the contribution of the maternal gut microbiota (and microbiota-generated metabolites) in determining fetal outcomes is largely unexplored. Here, we show that maternal gut communities and specific microbiota members are key modulators of placental phenotype with important consequences for fetal development. We have revealed novel roles for a maternal Bifidobacterium strain, that include the control of placental capillary morphogenesis and nutrient transporters (glucose and lipids), which affect fetal metabolism and growth. Our work has important implications for the establishment of novel therapeutic strategies to treat pregnancy complications.

\section{Abstract}

The gut microbiota plays a central role in regulating host metabolism. However, while substantial progress has been made in discerning how the microbiota influences host functions post birth and beyond, little is known about how key members of the maternal gut microbiota can influence feto-placental growth. Here, using germ-free and specific-pathogen-free mice, we demonstrate that the bacterium Bifidobacterium breve UCC2003 modulates maternal body adaptations, placental structure and nutrient transporter capacity, with implications for fetal metabolism and growth. The effects of B. breve UCC2003 on feto-placental growth are mediated, in part, by changes in the maternal and placental metabolome (i.e. acetate and carnitine). Histological analysis of the placenta confirmed that Bifidobacterium modifies placental structure via changes in Igf2P0, Dlk1, Mapk1 and Mapk14 expression. Additionally, $B$. breve UCC2003, acting through SIc2a1 and Fatp3-4 transporters, was shown to restore fetal glycaemia and fetal growth in association with changes in the fetal hepatic transcriptome. This study provides knowledge towards a novel and safe therapeutic strategy for treating pregnancy disorders via modulation of the maternal gut microbiota.

\section{Main Text}

\section{Introduction}

All nutrients and metabolites required for feto-placental growth are provided by the mother, which in turn is thought to be influenced by the maternal gut microbiota through breakdown of complex dietary components (1). During gestation, liberated metabolites may be used by the placenta for morphogenesis, and transported across the placenta for use by the fetus for growth and development $(2,3)$. This is highly important across gestation, particularly at later stages, when fetal growth is maximal. Notably, there are also alterations in the maternal microbiota throughout pregnancy with levels of the beneficial bacterial genus Bifidobacterium 
rising from trimester 1 onwards (4-6). Failure of the mother to provide nutrients and metabolites to the fetus can result in pregnancy complications including small for gestational age (SGA), fetal loss and stillbirth. However, the contribution of the maternal microbiota in determining fetal outcomes is largely unexplored. Knowledge in this area would be highly valuable for developing treatments to improve fetal growth, with benefits for population health.

Studies performed with Germ free (GF) mice have identified that the microbiota is a key regulator for adequate development, early immune education and metabolism (7-11). However, little is known about how maternal microbiota influences feto-placental growth and more specifically, placental structure and function. Here, we hypothesized that the maternal gut microbiota, and specific microbiota members, regulate fetal growth by modulating placental development and nutrient supply. We tested this hypothesis by comparing conceptus growth in three different maternal gut microbiota conditions by using conventional specific-pathogen-free (SPF) mice (used as a base line to define correct feto-placental growth) (11), GF mice, and maternal GF mice colonized with Bifidobacterium breve UCC2003 (12). As a keystone microbiota member and 'probiotic' species, B. breve may represent a suitable option for treating pregnancy complications by exerting beneficial metabolic effects on maternal physiology and associated feto-placental growth. Indeed, B. breve induced changes in maternal physiology, placental morphogenesis and the abundance of placental glucose and lipid transporters, which were associated with improvements in growth and metabolism of the fetus.

\section{Results}

\section{Germ-free mice treated with B. breve have altered body composition and caecum} metabolic profile

To assess whether maternal microbiota is able to influence feto-placental growth, GF mice were treated orally with B. breve UCC2003 from day 10 of gestation (treatment on days 10, 12 and 14; i.e. BIF group), and compared to GF and SPF dams (for experimental overview see Figure S1). Timing and dosing were based on the fact that levels of Bifidobacterium rise throughout pregnancy (5) (colonization levels during pregnancy can be found in Figure S2). Moreover, previous work has indicated three consecutive doses of B. breve UCC2003 facilitates stable gut colonization, with the advantage of also avoiding repeated handling of the mice, which may induce spontaneous abortions $(13,14)$. In addition, from a translational point of view, we also wanted to correlate with potential future supplementation studies in women at the point pregnancy is confirmed.

Maternal body composition differed between groups with GF and BIF mice showing increased digestive tract weight and lower pancreas mass compared to SPF mice. GF and BIF mice had similar circulating concentrations of glucose and insulin to SPF mice (assessed in fed 
conditions; Table 1). Compared to SPF mice, treatment with $B$. breve reduced maternal gonadal fat depot, liver, and spleen weights in BIF mice. No differences were observed in the circulating concentrations of leptin, cholesterol, triglycerides, or free fatty acids in maternal serum (Table 1).

Metabolomics analysis in maternal caecum samples indicated that the concentration of 13 out of 115 metabolites were significantly altered (Table 1 and Table S1). Acetate was significantly influenced by $B$. breve (Table 1), with BIF dams having intermediate concentrations compared to SPF and GF mice (the low levels of acetate detectable in GF mice, most likely originated from the diet and/or are host-derived). These findings suggest that acetate producing $B$. breve and the wider gut microbiota may exert selective effects on maternal metabolic (gonadal fat depot and liver) and immune organs (spleen).

\section{Maternal gut microbiota and $B$. breve regulate fetal growth by controlling fetal glycaemia}

Although the three experimental groups had similar numbers of viable fetuses per litter, the GF group had higher numbers of resorptions within the litter (defined as large or small hemorrhagic-to-black implantation sites) compared to SPF and BIF mice (Figure 1A-B). Similarly, when this parameter was calculated as resorption rate, and corrected for litter size, we found similar results (Figure S3). In fact, all GF dams had at least one resorption, whilst $B$. breve reduced the appearance of resorptions by $50 \%$ (Figure 1B). No evidence of intrauterine death, defined as a lack of fetal vascular flow in the yolk sac vessels, were found. Compared to SPF and BIF mice, GF fetuses were growth restricted, hypoglycaemic and had reduced liver weight, but had preserved brain size (Figure 1C-E). These findings highlight that the maternal microbiota influence fetus viability and that $B$. breve is a strong modulator of fetal growth and metabolism.

\section{Maternal B. breve modulates fetal hepatic transcriptome}

The liver is a key organ for glucose storage and production. As fetuses from BIF mice had enlarged livers and improved glycaemia, we next determined if there were changes in the fetal hepatic transcriptome (livers were collected on GD18.5, when fetal liver function is particularly active prior to term; Figure 1F). A total of 602 genes were differentially expressed, with 94 significantly up-regulated and 508 down-regulated genes in BIF group, when compared to GF group (Figure 1G-H). Functional enrichment analysis indicated many metabolic pathways were regulated in the fetal livers of BIF mice including carboxylic acid and lipid metabolic processes, steroid hydroxylase activity, fatty acid metabolism and response to glucocorticoid (Figure 1l; Table S2). Therefore, maternal $B$. breve appears to exert beneficial alterations in fetal hepatic function to support fetal growth. 


\section{Maternal gut microbiota and B. breve control placental morphogenesis}

To further understand the links between the maternal microbiota and the regulation of fetal growth, we assessed placental structure. When compared to SPF mice, placentas were lighter in GF and BIF mice (Figure 2A). Placental efficiency, defined as the grams of fetus produced per gram of placenta, was significantly improved in the BIF group compared to SPF and GF mice (Figure 2B). Analysis of placental compartments showed that lack of maternal gut microbiota significantly hampered growth of the placental labyrinth transport zone (Lz), without compromising the endocrine junctional zone or decidua volumes (Figure $2 \mathrm{C}$ ). It also did not affect placental glycogen storage (Figure 2D) or the volume of the trophoblast (Figure E-F). Analysis of maternal blood spaces revealed that the BIF group had reduced spaces compared to SPF mice, while the volume and the length of fetal capillaries were significantly reduced in the GF compared to SPF (Figure 2F-G). Similarly, surface area for exchange of the Lz was significantly decreased in GF compared to SPF mice (Figure $2 \mathrm{H}$ ). The barrier between maternal and fetal blood was also determined to be thinner in BIF versus GF mice (Figure 2I). Lz apoptosis levels were similar between groups (Figure $2 \mathrm{~J}$ ).

To define the molecular mechanisms behind the changes in the Lz, we quantified the expression of select genes in micro-dissected Lz. Common angiogenic factors Vegf, Ang1, Ang2 were similarly expressed between groups (Figure 2K). However, the expression of signaling pathways involved in cell proliferation and growth, namely the MAPK pathway, was significantly altered by changes in maternal gut microbiota; Mapk1 was shown to be increased in both GF and BIF, while Mapk14 (also known as p38Mapk) was revealed to be specifically up-regulated in the Lz of BIF mice. In addition, DIk1 and Igf2PO, which are key genes implicated in metabolism and Lz formation, were significantly up-regulated in the BIF group compared to GF mice. The expression of Akt did not vary with group (Figure 2K). As informed by western blotting, activation of ERK was reduced in the placental Lz of GF compared to SPF mice, and this effect was reversed by BIF (Figure 2L). p38MAPK protein activity was similar between groups. DLK1 protein level was also lower in GF compared to SPF mice. However BIF increased DLK1 protein levels when compared to both SPF and GF mice (Figure 2L). Further analysis by qPCR showed that the mRNA levels of Nras, but not Kras, were significantly elevated in the BIF compared to the SPF group (Figure 2M). Overall, these findings suggest that the maternal gut microbiota, and $B$. breve, regulate the development of the mouse placental Lz via modulation of specific cell growth and metabolic genes/pathways.

Maternal gut microbiota and B. breve affect placental glucocorticoid handling and controls key placental nutrient transporters

Glucocorticoids are a class of steroid hormones that can affect both fetal and placental formation. To control glucocorticoid actions on the conceptus, the placenta expresses $11 \beta$ hydroxysteroid dehydrogenase type 1 and 2 (11Hsd1 and 11Hsd2). We found that the 
expression of $11 H s d 1$ was significantly elevated in the BIF group compared to the GF (no change in $11 \mathrm{Hsd}$ 2; Figure $2 \mathrm{~N}$ ). Moreover, to better understand the changes in fetal growth and glycemia between groups, we quantified the expression of selected amino acid, glucose and lipid transporters in the Lz. We found no difference in the expression of system A amino acid transporters (S/c38a1, S/c38a2, S/c38a4) between groups (Figure 2O). However, the key glucose transporter SIc2a1 was up-regulated in the Lz of BIF mice compared to GF mice (Slc2a3 mRNA levels were similar between groups; Figure 2O). Fatty acid transporters were also altered, with high expression levels of Fatp1 in the GF versus the SPF group, and increased levels of Fatp3 and Fatp4 in the BIF group compared to the GF (Figure 2O; Cd36 expression levels were unaltered). Collectively, these data suggest that maternal gut microbiota, and $B$. breve, regulate fetal growth by inducing changes in the expression of key nutrient transporters within the placenta.

\section{Differences in placental labyrinth growth are linked to an altered placental metabolome}

To gain further mechanistic understanding of the changes observed in the placental Lz and fetal liver, we analysed $>80$ metabolites at GD16.5 (Figure 3 and Table S1). We found 5 metabolites significantly altered in the placental Lz (Figure 3). Aminoadipic acid in the Lz was very low in GF/BIF groups as well as in fetal livers (Figure $3 A$ ). Treatment with $B$. breve significantly reduced the concentrations of acetylcarnitine and carnitine in Lz tissue compared to SPF placentas, but not in fetal livers (Figure 3B-C). Levels of formate in placental Lz were significantly elevated in both GF and BIF compared to SPF mice (Figure 3D), with a similar trend (although not significant) in fetal liver samples. Acetate was also altered in the Lz (Figure $3 \mathrm{E}$ ), with concentrations significantly lower in the SPF compared to the GF group, whilst BIF samples showed intermediate levels (although these levels were much lower than observed in the maternal caecum). Similar to formate, concentrations of acetate in fetal liver followed similar directions to the Lz, yet were not statistically different between groups. These data suggest that maternal gut microbiota, and $B$. breve, regulate the fetal and placental growth via modulation of the placental Lz metabolome.

\section{Discussion}

In this study, we provide evidence that the maternal microbiota and the microbiota member $B$. breve regulate maternal body composition during gestation and beneficially modulate fetoplacental growth. To the best of our knowledge, this is the first demonstration of a maternal gut bacterium remotely controlling placental structure and nutrient transporters, with important implications for fetal glycaemia and fetal growth. We observed that the effects of Bifidobacterium are partially mediated by altered metabolites in maternal caecum and in placental Lz tissue, with alterations in the expression of key genes in the placental Lz and fetal liver. 
Bifidobacterium is the dominant microbiota member in vaginally delivered, breast fed infants, with certain species and strains known to stimulate and aid the maturation of the immune system (15). B. breve UCC2003 also regulates responses at the gut barrier, inducing homeostatic epithelial cell programming, and protecting against inflammatory insults $(14,16)$. Importantly, pregnancy is accompanied by increasing Bifidobacterium abundance in the gut of women and mice (5), which is associated with changes in progesterone concentrations during pregnancy. Additionally, alterations in the abundance of Bifidobacterium are also linked to the development of serious pregnancy complications like preeclampsia (17). Our study shows that $B$. breve improves pregnancy outcomes in mice, as indicated by the lower number of resorptions, elevated placental efficiency and restored fetal glycaemia and weight. $B$. breve UCC2003 was also shown to induce changes in the metabolite milieu, including acetate, acetylcarnitine and carnitine in the mother and placenta, which were likely key for the beneficial effects on fetal growth. Acetate is a major bifidobacterial fermentation by-product, which directly mediates epithelial cell responses but also exerts systemic beneficial metabolic effects $(18,19)$. More generally, microbial-derived short-chain fatty acids (SCFAs) modulate multiple host physiological systems and during pregnancy are associated with maternal gestational weight, neonatal length and body weight, and protection against allergic airway disease in the developing fetus $(20,21)$. Acetate crosses the placenta $(21)$, so in our model, the elevated maternal $B$. breve-derived acetate may exert beneficial effects on feto-placental growth via (i) maternal effects, through interactions within the maternal gut mucosa as evidenced by higher maternal caecum acetate concentrations in $B$. breve supplemented (and SPF dams vs. GF) and the subsequent impact on maternal organs (liver, adipose and spleen) and nutrient handling, (ii) effects on the placenta, through use of acetate for cellular metabolism, growth and function, and/or (iii) effects on the fetal metabolism following transport of acetate across the placenta to the fetus. Compared to the maternal caecum, levels of acetate were relatively low in the placental Lz and fetal liver (for all 3 groups). This suggests that $B$. breve (and SPF microbiota-derived acetate) may be used to support anabolic processes in utero (hence the very low levels detected), and together with changes in acetylcarnitine and carnitine, may be supported by our observations of elevated fatty acid transporter expression in the placenta and differentially regulated genes in the fetal liver. The observed modulation of immune-associated pathways in the fetal liver, including those associated with G protein-coupled receptor signalling (e.g. Dusp9), also indicates a role for direct acetate-associated responses (22). B. breve supplementation also restored fetal glycaemia and weight in BIF mice, achieving similar values to that seen for SPF fetuses. Previous in vivo studies indicate different strains of Bifidobacterium (including $B$. breve) modulate glucose handling (23), with this genus also consistently associated with potential protection against human metabolic disorders e.g. type 2 diabetes $(24,25)$. In addition, our observations of reduced maternal gonadal fat mass and maternal liver weight in $B$. brevetreated dams compared to SPF dams, suggest that Bifidobacterium, or B. breve metabolites, 
could affect maternal metabolic responses in key organs of the mother. In fact, previous work in adult mice suggests elevation of gut acetate levels due to Bifidobacterium treatment plays a key role in regulating glucose handling systemically and reduces visceral fat accumulation (26).

Maternal microbiota significantly affected the placental vascular bed by modifying the elongation of their fetal capillary network, which is required for adequate fetal growth (27). Moreover, $B$. breve significantly reduced the interhaemal membrane barrier thickness of the placenta, which may facilitate exchange of nutrients and gases. The mechanisms governing these structural changes could be partially mediated by changes in the expression of two imprinted genes (28), Igf2 (namely the placenta-exclusive isoform, Igf2P0) and Dlk1, and via MAPK upregulation. In this regard, deletion of Igf2PO results in feto-placental growth restriction in association with reduced placental surface area for exchange, fetal capillary volume and increased barrier thickness (reviewed by (29)); parameters that were partially improved by $B$ breve UCC2003 in line with the Igf2PO mRNA upregulation. Interestingly, Igf2PO is important for the structural and transport adaptations occurring in the placenta in response to maternal nutritional restriction (30) and recent work has shown that lgf2 in the placenta interacts with MAPK signaling (31). DIk1, a non-canonical ligand of the Notch signalling pathway localized to the endothelial cells of fetal capillaries in the placental Lz, also regulates placental vascularisation and branching morphogenesis (32). In addition, IGF2 and DLK1 can both mediate cellular actions via the MAPK pathway $(33,34)$. Mapk14, also known as p38MAPK, forms part of a signal transduction pathway that has been linked to environmental stresses and inflammatory cytokines (35). However, p38MAPK also regulates many normal cellular processes, including proliferation and cytoskeletal organisation. Furthermore, research in mice has demonstrated that Mapk14 is essential for embryogenesis and placental Lz angiogenesis and vascular remodeling (36). In addition, placentas exposed to B. breve UCC2003, had lower concentrations of acetylcarnitine and carnitine compared to SPF, suggesting a greater reliance on these compounds for energy production, or enhanced transfer of these fatty acids to the fetus (37). Carnitine is well-known for mediating the transport of fatty acids into mitochondrial matrix for fatty acid $\beta$-oxidation. Carnitine can also promote p38MAPK signalling activation in cardiac tissue (38). Taken together, placental structural, transport and metabolic changes seen with $B$. breve UCC2003 treatment may link to the altered metabolites/nutrient milieu in the mother and would have been beneficial in providing additional substrates for fetal growth and development (when compared to GF; Figure S4).

While our study has clear strengths and strong translational implications for pregnancy complication treatments, it also has certain limitations. Unsurprisingly, we did not see a full 'rescue' of placental phenotype in the monocolonised GF B. breve (BIF) group, compared to complex microbiota found in SPF dams. However, structural and functional adaptations of 
these placentas exposed to $B$. breve were adequate enough to 'rescue' fetal weight and fetal glycaemia. An array of gut-associated signaling and a diverse metabolite pool are expected to provide more complete placental development. Indeed, other or additional Bifidobacterium species and/or strains may be required for placental and fetal development, given strainspecific host physiology responses $(16,39)$. Further studies should allow the relative contributions of other microbial- and Bifidobacterium-derived factors to be elucidated. In addition, future work should evaluate the role of $B$. breve across gestation using larger cohorts of mice. Nonetheless, this study has revealed novel roles for Bifidobacterium and provides a safe therapeutic strategy for treating pregnancy complications, suggesting an opportunity for in utero programming through maternal Bifidobacterium and associated metabolites.

Overall, although our study was performed in mice and is not representative of a clinical scenario, our study highlights the importance of the maternal gut microbiota during gestation and demonstrates that $B$. breve modulates maternal physiology, placental structure and nutrient transporter capacity with impact on fetal glycaemia and fetal growth (Figure S4). Our findings prompt an in-depth investigation into how additional members of the gut microbiota impact on pregnancy outcomes. These future studies are important for the design of novel therapies to combat fetal growth restriction and other pregnancy complications.

\section{Materials and Methods}

\section{Bifidobacterium breve UCC2003/pCheMC}

$B$. breve UCC2003/pCheMC was generated by introducing the plasmid pCheMC to electrocompetent B. breve UCC2003 as described previously (40). In brief, B. breve UCC2003 was grown until mid-log phase, chilled on ice and washed twice with ice cold sucrose citrate buffer ( $1 \mathrm{mM}$ citrate, $0.5 \mathrm{M}$ sucrose, pH5.8) and then electroporation of cells was carried out under the following conditions; $25 \mathrm{MF}, 200 \mathrm{Ohms}, 2 \mathrm{KV}$. Transformed cells were incubated for 2 hours in Reinforced Clostridial Medium (RCM) at $37^{\circ} \mathrm{C}$ in a controlled anaerobic chamber then plated (41) on RCM agar plates with selective antibiotics. Colonies were sub-cultured 3 times on RCM agar plates with selective antibiotics. Antibiotics were used at the following final concentrations erythromycin $2 \mu \mathrm{g} / \mathrm{mL}$.

\section{Lyophilised $B$ breve}

$B$. breve was grown in De Man, Rogosa and Sharpe agar (MRS) under anaerobic conditions overnight. The bacterial cell pellet was resuspended in $10 \%$ milk powder and lyophilised in 200

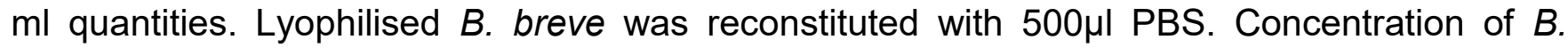
breve was $10^{10} \mathrm{CFU} / \mathrm{ml}$. All batches were tested for contamination upon reconstitution on LuriaBertani (LB) and Brain-Heart Infusion (BHI) plates under anaerobic and aerobic conditions at $37^{\circ} \mathrm{C}$. No contamination of $B$. breve was detected. 


\section{Mice}

All mouse experiments were performed under the UK Regulation of Animals (Scientific Procedures) Act of 1986. The project license PDADA1B0C under which these studies were carried out was approved by the UK Home Office and the UEA Ethical Review Committee. All mice were housed in the Disease Modelling Unit at the University of East Anglia, UK. Animals were housed in a 12:12 hour light/dark, temperature-controlled room and allowed food and water ad libitum. Female Germ Free C57BL/6J (GF) and Specific Pathogen Free (SPF) mice aged 6-8 weeks were used for the study. GF mice were bred in germ free isolators (2 females to 1 male) and on gestational day (GD) GD9.5, pregnant mice (confirmed by weight) were removed from the GF isolator and transferred to individually ventilated cages. The sterility of these cages was previously tested and found to be suitable for housing GF mice for 1 week. Sterile water was changed every 2 days.

\section{B. breve colonisation levels}

Mice were given $100 \mu \mathrm{L}$ of reconstituted lyophilised $B$. breve UCC2003 by oral gavage (containing $10^{10} \mathrm{CFU} / \mathrm{mL}$ ) at GD10, GD12 and GD14 or $100 \mu \mathrm{L}$ vehicle control (PBS, $4 \%$ skimmed milk powder), with this dosing regimen reflecting a more realistic time frame for women who are more likely to start taking probiotics once their pregnancy is confirmed. At GD16.5 and GD18.5, mice were sacrificed by cervical dislocation and samples collected for molecular and histological analysis. The experimental design can be found in Figure S1.

Faecal samples were checked for contamination and B. breve colonization at GD12 and GD14 and GD16. Briefly, faecal samples from GF and GF treated with B. breve were diluted in 500 $\mu \mathrm{L}$ of PBS and agitated for 30 mins at $4^{\circ} \mathrm{C}$ on an Eppendorf MixMate 5353 Digital Mixer Plate Shaker. The faecal solution was passed through a $0.45 \mu \mathrm{m}$ syringe filter. Faecal solution was diluted 1 in 100 and $20 \mu \mathrm{L}$ was added to a De Man, Rogosa and Sharpe agar plate with erythromycin and incubated for 48 hours in an anaerobic chamber at $37^{\circ} \mathrm{C}$. Colony forming units were counted using a click counter.

\section{Blood hormones and circulating metabolites}

Maternal blood was obtained by cardiac exsanguination immediately after cervical dislocation. Blood was centrifuged and serum collected and stored at $-80^{\circ} \mathrm{C}$ until further analysis. Blood glucose and plasma concentrations of leptin, insulin, triglycerides, cholesterol, and free fatty acids were determined as previously reported (42). Fetal blood glucose levels were measured with a handheld glucometer (One Touch Ultra; LifeScan) immediately after decapitation of the fetus.

\section{Placental histology}

Placentas were cut in half and fixed in $4 \%$ paraformaldehyde overnight at $4^{\circ} \mathrm{C}$. Samples were 
washed 3 times with PBS for 15 minutes each and storage in 70\% ethanol until embedding in wax. Embedded placentas were cut at $5 \mu \mathrm{m}$ thickness and stained with haematoxylin and eosin for gross morphology. Placental layer volumes were calculated using the Computer Assisted Stereological Toolbox (CAST v2.0). For analysis of labyrinth components, sections were stained with lectin for identification of fetal endothelial vessels and with cytokeratin for trophoblasts. Further details of the double-labelling immunohistochemistry and the analysis performed can be found elsewhere (43). For the analysis of placental glycogen, sections were stained with Periodic acid-Schiff (Sigma-Aldrich) previous incubation with $0.5 \%$ periodic acid (Thermo Fisher Scientific). Sections were counterstained with Fast-green (Sigma-Aldrich) and digitalized with the nanozoomer scanner (Hamamatsu). Analysis of placental glycogen accumulation was performed with Image $\mathrm{J}$ and conducted blinded to experimental groups. TUNEL staining for placental cell death was performed using the TUNEL Assay Kit - HRP-DAB (Abcam, ab206386) following manufacturer instructions except for the counterstaining which was substituted for Nuclear Fast Red (Vector). Sections were digitalized using a nanozoomer scanner (Hamamatsu) and the amount of apoptosis in the labyrinth zone was calculated in 5 random areas (x20 magnification) and analysed by Image $\mathrm{J}$ software.

\section{Western blotting}

Protein extraction was performed with RIPA buffer as described previously (44). Lysates were separated by SDS-PAGE and incubated with antibodies against p-MAPK (Thr202/Tyr204) (Cell Signalling, 4370; 1/1000), t-MAPK 44/42 (Cell Signalling, 4695; 1/1000), DLK-1 antibody (Abcam, ab21682; 1/1000), p-P38MAPK (Cell Signalling, 4511; 1/1000) and t-P38MAPK (Cell Signalling, 8690; 1/1000). Reactive bands were detected by chemiluminescence (Thermo Scientific, Scientific SuperSignal West Femto) and quantified by Image J software. Proteins were normalized to Ponceau S Staining (45).

\section{RNA extraction and qPCR}

Extraction of RNA from micro-dissected placental labyrinth zones was performed with RNeasy Plus Mini Kit (Qiagen) and reverse transcribed using the High Capacity cDNA RT Kit minus RT inhibitor (Applied Biosystems) according to manufacturer's instructions. Samples were analysed using MESA Blue SYBR (Eurogentec) and primers (See Supplementary Table 3) were synthesized by Sigma-Aldrich. The expression of each gene was normalized to the geometric mean expression of two reference genes Hprt and Ubc, which remained stably expressed across the groups. Analysis was performed using the $2-\Delta \Delta \mathrm{Ct}$ method (46).

\section{Sequence pre-processing, Differential Gene Expression (DGE) analysis and Functional enrichment analysis}

Fetal liver RNA on GD18.5 was extracted using the RNeasy Plus Mini Kit (Qiagen). RNA sequence pre-processing and DGE analysis was performed as previously described with slight 
modifications (14). Briefly, FASTQ reads were initially quality-filtered using fastp v0.20.0 with options -q 10 (sequence reads with phred quality <10 were discarded). Subsequently, sequence reads for each sample were merged (merge-paired-reads.sh) and followed by rRNA sequence filtering via SortMeRNA v2.1 based on SILVA rRNA database optimised for SortMeRNA software $(47,48)$. Filtered reads were then unmerged (unmerge-paired-reads.sh) and ready for transcript quantification. Transcript mapping and quantification were then performed using Kallisto v0.44.0 (49). Mus musculus (C57BL/6 mouse) cDNA sequences (GRCm38.release-98_k31) retrieved from Ensembl database were indexed with Kallisto utility index at default parameter and was used for following transcript mapping and abundance quantification via Kallisto utility quant at 100 bootstrap replicates (-b 100) (50).

RNA raw counts were subjected (Kallisto outputs) to DGE analysis, which was performed using R library Sleuth (v0.30.0) (51). Transcripts were then mapped to individual genes using Ensembl BioMart database (GRCm38.p6) with function sleuth_prep and option gene_mode = TRUE. Genes with an absolute $\log _{2}$ (fold change) $>1.0$ and $q$ value $<0.05$ ( $p$-adjusted value; based on Wald test statistics) were considered to be differentially regulated (52). DGE statistics were plotted via functions within package Sleuth. Finally, functional enrichment analysis was performed using g:Profiler webtool g:GOst based on organism Mus Musculus species (53). Briefly, a list of DGEs (Ensembl IDs) was uploaded to g:GOst, then selected 'GO molecular function', 'GO biological process' and 'Reactome' in the 'data sources'. Significance threshold was set at 0.001 (g:SCS threshold).

\section{Metabolite extraction, Nuclear Magnetic Resonance (NMR) spectroscopy and metabolite} quantification

Extraction of metabolite from liver, placenta, caecum contents and culture (spent) media samples were performed as previously described as a standard protocol (54). For caecal samples, frozen materials (stored at $-80^{\circ} \mathrm{C}$ prior to analysis) were weighed $\sim 50 \mathrm{mg}$ before the addition of $600 \mu \mathrm{L}$ of faecal water phosphate buffer solution. The faecal water phosphate buffer

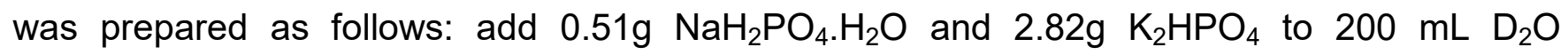
(Deuterium Oxide; Merck). To this, $34.5 \mathrm{mg}$ TSP (Trimethylsilyl propanoic acid; used as NMR standard) and $100 \mathrm{mg} \mathrm{NaN}_{3}$ (Merck) were added (55). Next, the mixture was centrifuged for 10 min at $17,000 \times \mathrm{g}$ before transferring the mixture to an NMR tube (Merck) for subsequent NMR analysis.

For liver and placenta samples (stored at $-80^{\circ} \mathrm{C}$ prior to analysis), frozen fresh tissue ( 20-45 $\mathrm{mg}$ ) was placed into a $2 \mathrm{ml}$ sterile microcentrifuge tube pre-loaded with $\sim 15-20$ glass beads (Merck) while $200 \mu \mathrm{L}$ of ice-cold methanol (Fisher Scientific) and $42.5 \mu \mathrm{L}$ of ultra-pure cold water were added to it and vortexed. Tissue was disrupted via a tissue lyser (Qiagen) for $2 \times 2$ mins. $100 \mu \mathrm{L}$ of ice-cold chloroform (Merck) was then added and vortexed. $100 \mu \mathrm{L}$ of ice-cold 
chloroform and $100 \mu \mathrm{L}$ of ultra-pure cold water were added to the mixture, and kept on ice for $15 \mathrm{~min}$. Liquid was then transferred into a new sterile microcentrifuge tube and centrifuged for $3 \mathrm{~min}$ at $17,000 \times \mathrm{g}$. The top aqueous phase was transferred into a new microcentrifuge tube and speed-vacuumed for $30 \mathrm{~min}$ at $50^{\circ} \mathrm{C}$ and $30 \mathrm{~min}$ without heating prior to reconstitution with faecal water phosphate buffer solution at $600 \mu \mathrm{L}$. The mixture was then moved to an NMR tube (Merck) for subsequent NMR analysis. Metabolites from culture media Brain Heart Infusion (BHI; Oxoid) and spent media (BHI cultured with B. breve UCC2003 for $48 \mathrm{~h}$ ) were extracted as follows: $400 \mu \mathrm{L}$ of medium was transferred into a sterile microcentrifuge tube with the addition of $200 \mu \mathrm{L}$ faecal phosphate buffer and mixed well. The mixture was then moved to an NMR tube (Merck) for further NMR analysis.

Samples in NMR tubes were subsequently subjected to NMR spectroscopy. The ${ }^{1} \mathrm{H}$ NMR spectra were recorded at $600 \mathrm{MHz}$ on a Bruker AVANCE spectrometer (Bruker BioSpin $\mathrm{GmbH}$, Germany) running Topspin 2.0 software. The metabolites were then quantified using the software Chenomx® NMR Suite $7.0^{\mathrm{TM}}$.

\section{Statistical analysis}

All statistical analysis and sample size are shown in each figure/table and in the corresponding figure/table legends. Only samples from viable fetuses were analysed. No statistical analysis was used to pre-determine sample size and samples were assigned code numbers and analysis was performed in a blinded fashion. Statistical calculations were performed using the GraphPad Prism software (GraphPad, San Diego, CA), SAS/STAT (Statistical System Institute Inc. Cary, NC, USA) and RStudio Version 1.4.1106 (RStudio Boston, MA) with R Version 4.0.3 (Vienna, Austria). Morphometric parameters of mother and number of viable/resorption per litter were analysed by one-way ANOVA followed by Tukey post hoc test. Resorption rate was calculated with a general linear model with total litter size as a covariate. Feto-placental weights, placental stereological measurements and placental Lz gene expression levels were analysed with a general linear mixed model, taking into account viable litter size as a covariate and taking each fetus as a repeated measure. Differences between individual metabolites between the three groups were tested with a Kruskal-Wallis test using the kruskal.test function with correction for multiple comparisons applied using the Benjamini \& Hochberg false discovery rate method using the p.adjust function. Pairwise comparisons between the three groups were carried out with a Dunn's test on individual metabolites significantly different after correction for multiple comparisons using the dunnTest function in the FSA package. ROUT test was used for identification of outlier values. A probability value of $<0.05$ was considered significantly different.

\section{Acknowledgments}


JL-T currently holds a Sir Henry Wellcome Postdoctoral Fellowship (220456/Z/20/Z) and previously a Newton International Fellowship from the Royal Society (NF170988 / RG90199). LJH is supported by Wellcome Trust Investigator Awards (100974/C/13/Z and 220876/Z/20/Z); the Biotechnology and Biological Sciences Research Council (BBSRC), Institute Strategic Programme Gut Microbes and Health (BB/R012490/1), and its constituent projects BBS/E/F/000PR10353 and BBS/E/F/000PR10356. ANS-P is supported by a Lister Institute of Preventative Medicine Research Prize (RG93692). Authors would like to thank Dr Ruben Bermejo-Poza (Complutense University of Madrid) for statistical advice and the FergusonSmith laboratory (University of Cambridge) for providing the DLK1 antibody.

\section{Data availability}

The fetal liver RNA-Seq raw sequencing data are deposited at the National Center for Biotechnology Information (NCBI) under BioProject PRJNA748000. Relevant data are within the manuscript and its Supporting Information files. Scripts for differential gene expression analysis can be accessed at GitHub, https://github.com/raymondkiu/Maternal-foetalmicrobiota-paper/

\section{References}

1. R. Krajmalnik-Brown, Z.-E. Ilhan, D.-W. Kang, J. K. DiBaise, Effects of Gut Microbes on Nutrient Absorption and Energy Regulation. Nutr Clin Pract 27, 201-214 (2012).

2. B. McDonald, K. D. McCoy, Maternal microbiota in pregnancy and early life. Science 365, 984985 (2019).

3. M. G. de Agüero, et al., The maternal microbiota drives early postnatal innate immune development. Science 351, 1296-1302 (2016).

4. O. Koren, et al., Host remodeling of the gut microbiome and metabolic changes during pregnancy. Cell 150, 470-480 (2012).

5. M. Nuriel-Ohayon, et al., Progesterone Increases Bifidobacterium Relative Abundance during Late Pregnancy. Cell Rep 27, 730-736.e3 (2019).

6. T. Napso, H. Yong, J. Lopez-Tello, A. N. Sferruzzi-Perri, The role of placental hormones in mediating maternal adaptations to support pregnancy and lactation. Front. Physiol. 9 (2018).

7. E. S. Lamousé-Smith, A. Tzeng, M. N. Starnbach, The intestinal flora is required to support antibody responses to systemic immunization in infant and germ free mice. PLoS One 6, e27662 (2011).

8. E. A. Kennedy, K. Y. King, M. T. Baldridge, Mouse Microbiota Models: Comparing Germ-Free Mice and Antibiotics Treatment as Tools for Modifying Gut Bacteria. Frontiers in Physiology 9, 1534 (2018).

9. J. Lu, et al., Microbiota influence the development of the brain and behaviors in C57BL/6J mice. PLoS One 13, e0201829 (2018).

10. A. M. Martin, et al., The gut microbiome regulates host glucose homeostasis via peripheral serotonin. PNAS 116, 19802-19804 (2019).

11. M. M. Faas, et al., Microbiota Induced Changes in the Immune Response in Pregnant Mice. Front Immunol 10, 2976 (2019).

12. K. Pokusaeva, G. F. Fitzgerald, D. van Sinderen, Carbohydrate metabolism in Bifidobacteria. Genes Nutr 6, 285-306 (2011). 
13. S. Fanning, et al., Bifidobacterial surface-exopolysaccharide facilitates commensal-host interaction through immune modulation and pathogen protection. PNAS 109, 2108-2113 (2012).

14. R. Kiu, et al., Bifidobacterium breve UCC2003 Induces a Distinct Global Transcriptomic Program in Neonatal Murine Intestinal Epithelial Cells. iScience 23, 101336 (2020).

15. M. J. Dalby, L. J. Hall, Recent advances in understanding the neonatal microbiome. F1000Res 9 , F1000 Faculty Rev-422 (2020).

16. K. R. Hughes, et al., Bifidobacterium breve reduces apoptotic epithelial cell shedding in an exopolysaccharide and MyD88-dependent manner. Open Biol 7, 160155 (2017).

17. T. Miao, et al., Decrease in abundance of bacteria of the genus Bifidobacterium in gut microbiota may be related to pre-eclampsia progression in women from East China. Food \& Nutrition Research (2021) https:/doi.org/10.29219/fnr.v65.5781 (July 5, 2021).

18. S. Fukuda, et al., Bifidobacteria can protect from enteropathogenic infection through production of acetate. Nature 469, 543-547 (2011).

19. M. A. González Hernández, E. E. Canfora, J. W. E. Jocken, E. E. Blaak, The Short-Chain Fatty Acid Acetate in Body Weight Control and Insulin Sensitivity. Nutrients 11, 1943 (2019).

20. M. Priyadarshini, et al., Maternal short-chain fatty acids are associated with metabolic parameters in mothers and newborns. Transl Res 164, 153-157 (2014).

21. A. N. Thorburn, et al., Evidence that asthma is a developmental origin disease influenced by maternal diet and bacterial metabolites. Nat Commun 6, 7320 (2015).

22. C. H. Kim, Control of lymphocyte functions by gut microbiota-derived short-chain fatty acids. Cell Mol Immunol 18, 1161-1171 (2021).

23. K. Kikuchi, M. Ben Othman, K. Sakamoto, Sterilized bifidobacteria suppressed fat accumulation and blood glucose level. Biochem Biophys Res Commun 501, 1041-1047 (2018).

24. $\mathrm{H}$. Wu, et al., Metformin alters the gut microbiome of individuals with treatment-naive type 2 diabetes, contributing to the therapeutic effects of the drug. Nat Med 23, 850-858 (2017).

25. A. Solito, et al., Supplementation with Bifidobacterium breve BR03 and B632 strains improved insulin sensitivity in children and adolescents with obesity in a cross-over, randomized doubleblind placebo-controlled trial. Clinical Nutrition 40, 4585-4594 (2021).

26. R. Aoki, et al., A proliferative probiotic Bifidobacterium strain in the gut ameliorates progression of metabolic disorders via microbiota modulation and acetate elevation. Sci Rep 7, 43522 (2017).

27. J. López-Tello, et al., Fetal and trophoblast PI3K p110 a have distinct roles in regulating resource supply to the growing fetus in mice. Elife 8 (2019).

28. J. M. Frost, G. E. Moore, The Importance of Imprinting in the Human Placenta. PLOS Genetics 6, e1001015 (2010).

29. A. N. Sferruzzi-Perri, I. Sandovici, M. Constancia, A. L. Fowden, Placental phenotype and the insulin-like growth factors: resource allocation to fetal growth. J Physiol 595, 5057-5093 (2017).

30. A. N. Sferruzzi-Perri, et al., Placental-specific Igf2 deficiency alters developmental adaptations to undernutrition in mice. Endocrinology 152, 3202-3212 (2011).

31. I. Sandovici, et al., "The Imprinted Igf2-Igf2r Axis is Critical for Matching Placental Microvasculature Expansion to Fetal Growth" (2021).

32. A. Yevtodiyenko, J. V. Schmidt, Dlk1 expression marks developing endothelium and sites of branching morphogenesis in the mouse embryo and placenta. Developmental Dynamics 235, 1115-1123 (2006).

33. C.-C. Huang, et al., Soluble delta-like 1 homolog (DLK1) stimulates angiogenesis through Notch1/Akt/eNOS signaling in endothelial cells. Angiogenesis 21, 299-312 (2018).

34. K. Forbes, M. Westwood, P. N. Baker, J. D. Aplin, Insulin-like growth factor I and II regulate the life cycle of trophoblast in the developing human placenta. American Journal of Physiology-Cell Physiology 294, C1313-C1322 (2008). 
35. A. Cuenda, S. Rousseau, p38 MAP-Kinases pathway regulation, function and role in human diseases. Biochimica et Biophysica Acta (BBA) - Molecular Cell Research 1773, 1358-1375 (2007).

36. J. S. Mudgett, et al., Essential role for $\mathrm{p} 38 \mathrm{\alpha}$ mitogen-activated protein kinase in placental angiogenesis. PNAS 97, 10454-10459 (2000).

37. A. N. Sferruzzi-Perri, J. S. Higgins, O. R. Vaughan, A. J. Murray, A. L. Fowden, Placental mitochondria adapt developmentally and in response to hypoxia to support fetal growth. Proc. Natl. Acad. Sci. U.S.A. 116, 1621-1626 (2019).

38. Z. Fan, Y. Han, Y. Ye, C. Liu, H. Cai, I-carnitine preserves cardiac function by activating p38 MAPK/Nrf2 signalling in hearts exposed to irradiation. European Journal of Pharmacology 804, 712 (2017).

39. L. Ruiz, S. Delgado, P. Ruas-Madiedo, B. Sánchez, A. Margolles, Bifidobacteria and Their Molecular Communication with the Immune System. Front Microbiol 8, 2345 (2017).

40. A. Mazé, M. O'Connell-Motherway, G. F. Fitzgerald, J. Deutscher, D. van Sinderen, Identification and Characterization of a Fructose Phosphotransferase System in Bifidobacterium breve UCC2003. Applied and Environmental Microbiology 73, 545 (2007).

41. M. Cronin, et al., High Resolution In Vivo Bioluminescent Imaging for the Study of Bacterial Tumour Targeting. PLOS ONE 7, e30940 (2012).

42. B. Musial, et al., Proximity to Delivery Alters Insulin Sensitivity and Glucose Metabolism in Pregnant Mice. Diabetes 65, 851-860 (2016).

43. K. De Clercq, J. Lopez-Tello, J. Vriens, A. N. Sferruzzi-Perri, Double-label immunohistochemistry to assess labyrinth structure of the mouse placenta with stereology. Placenta 94, 44-47 (2020).

44. E. Salazar-Petres, D. P. Carvalho, J. Lopez-Tello, A. N. Sferruzzi-Perri, "Placental mitochondrial function, nutrient transporters, metabolic signalling and steroid metabolism relate to fetal size and sex in mice" (2021).

45. I. Romero-Calvo, et al., Reversible Ponceau staining as a loading control alternative to actin in Western blots. Anal Biochem 401, 318-320 (2010).

46. K. J. Livak, T. D. Schmittgen, Analysis of relative gene expression data using real-time quantitative PCR and the 2(-Delta Delta C(T)) Method. Methods 25, 402-408 (2001).

47. S. Chen, Y. Zhou, Y. Chen, J. Gu, fastp: an ultra-fast all-in-one FASTQ preprocessor. Bioinformatics 34, i884-i890 (2018).

48. E. Kopylova, L. Noé, H. Touzet, SortMeRNA: fast and accurate filtering of ribosomal RNAs in metatranscriptomic data. Bioinformatics 28, 3211-3217 (2012).

49. N. L. Bray, H. Pimentel, P. Melsted, L. Pachter, Near-optimal probabilistic RNA-seq quantification. Nat Biotechnol 34, 525-527 (2016).

50. D. R. Zerbino, et al., Ensembl 2018. Nucleic Acids Res 46, D754-D761 (2018).

51. H. Pimentel, N. L. Bray, S. Puente, P. Melsted, L. Pachter, Differential analysis of RNA-seq incorporating quantification uncertainty. Nat Methods 14, 687-690 (2017).

52. R. J. Kinsella, et al., Ensembl BioMarts: a hub for data retrieval across taxonomic space. Database (Oxford) 2011, bar030 (2011).

53. U. Raudvere, et al., g:Profiler: a web server for functional enrichment analysis and conversions of gene lists (2019 update). Nucleic Acids Res 47, W191-W198 (2019).

54. G. Le Gall, Sample collection and preparation of biofluids and extracts for NMR spectroscopy. Methods Mol Biol 1277, 15-28 (2015).

55. J. Wu, Y. An, J. Yao, Y. Wang, H. Tang, An optimised sample preparation method for NMR-based faecal metabonomic analysis. Analyst 135, 1023-1030 (2010). 
bioRxiv preprint doi: https://doi.org/10.1101/2021.07.23.453407; this version posted November 14, 2021. The copyright holder for this preprint (which was not certified by peer review) is the author/funder, who has granted bioRxiv a license to display the preprint in perpetuity. It is made
A) Viable
B) Fetal available under aCC-BY-NC-ND 4.0 International license.
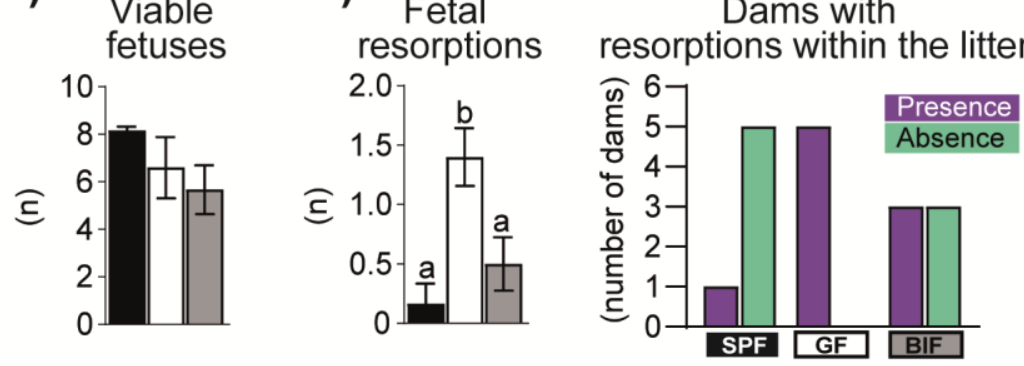
C) Fetal
D)

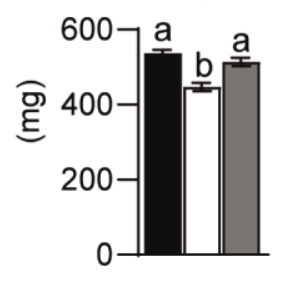
Fetal glycaemia

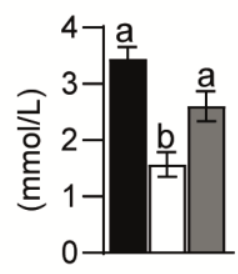

E)
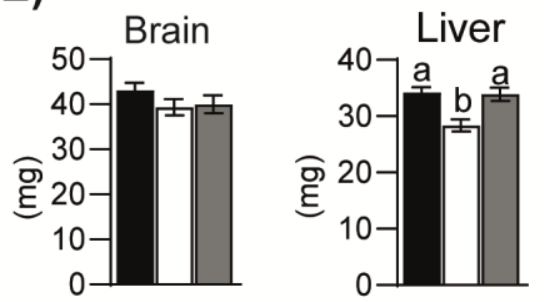

H) Top 20 dysregulated genes

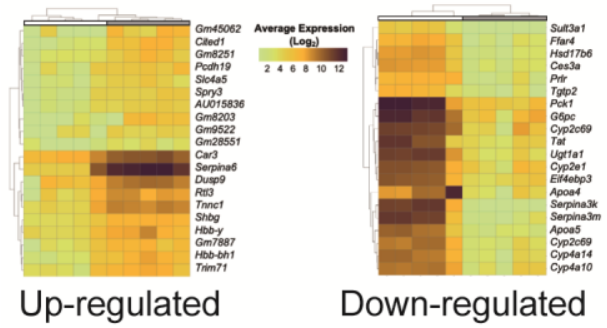

F)

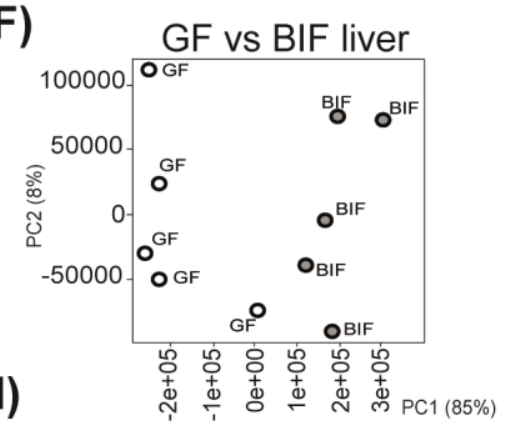

G)
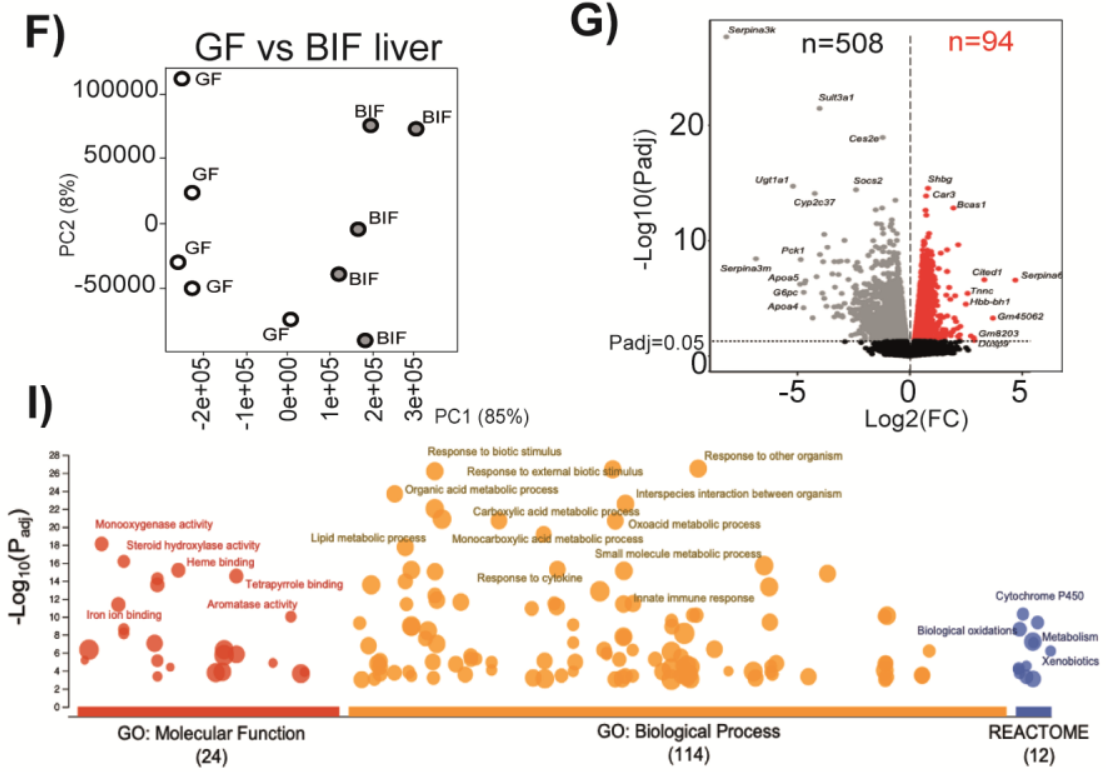

646

647

648

649

650

651

652

653

654

655

656

657

658

659

660

661

662

Figure 1. Effects of maternal gut microbiome and $B$. breve supplementation during pregnancy on fetal viability, growth and hepatic transcriptome. (A) Number of viable fetuses per litter, (B) Number of resorptions per litter (left) and number of dams with presence of resorptions within the litter (right), (C) Fetal weight, (D) Fetal blood glucose concentrations, (E) Fetal organ weights. All data shown in A to E were obtained on GD16.5 from: SPF (49 fetuses/6 dams), GF (33 fetuses/5 dams), BIF (34 fetuses/6 dams). (F-I) RNA-Seq analysis of fetal liver samples obtained at GD18.5, (F) PCA plot and (G) volcano plots showing up and down-regulated DEGs in BIF group (compared to GF group), (H) Heat map of the 20 most up and down-regulated DEGs (BIF group), (I) Functional profiling (g:Profiler) on 602 DEGs. Key enriched GO terms and REACTOME pathways are shown in the figure (significant threshold: $P_{\text {adj }}<0.001$ ). RNA-seq was performed on fetal livers obtained at GD18.5 from a total of $3 \mathrm{GF}$ and 4 BIF pregnant dams/litters. Statistical analysis for data shown in A-B was performed by one-way ANOVA Tukey multiple comparisons test. Data in C-E was analysed with a general linear mixed model, taking into account litter size as a covariate and taking each fetus as a repeated measure followed by Tukey multiple comparisons test. RNA-Seq data analysis is described in the material and methods section. Different superscripts indicate significant differences between groups (a versus $b$ and/or $c$ : $P<0.05$; mean $\pm S E M$ ). 
bioRxiv preprint doi: https://doi.org/10.1101/2021.07.23.453407; this version posted November 14, 2021. The copyright holder for this preprint (which was not certified by peer review) is the author/funder, who has granted bioRxiv a license to display the preprint in perpetuity. It is made A)

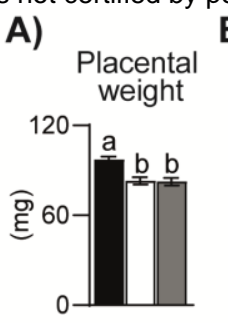

E)

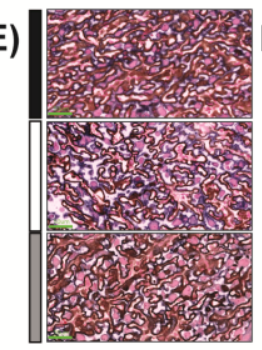

H) Surface I)

B) Placental

avadible under aCC-BY-NC-ND 4.0 International licenE9)

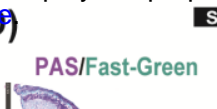

SPF GF

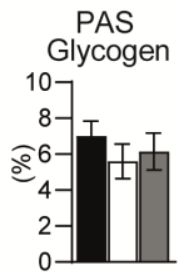

G)

F) Maternal blood

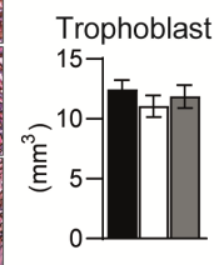

I) Barrier

J)

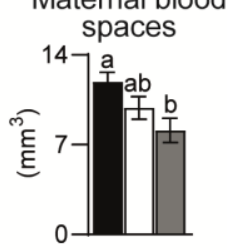

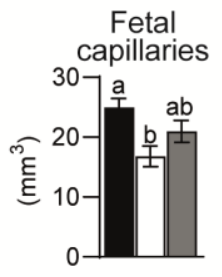
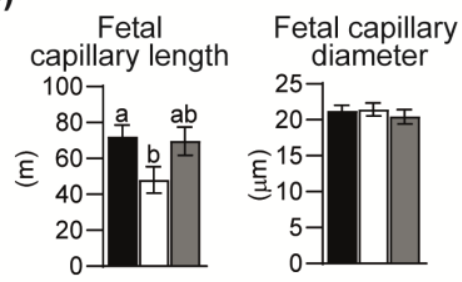

area for exchange
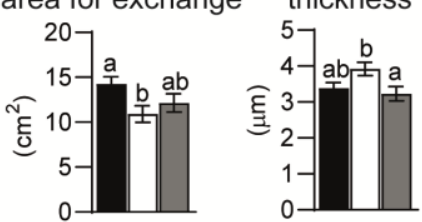

TUNEL DAB+/Fast-Red

K)
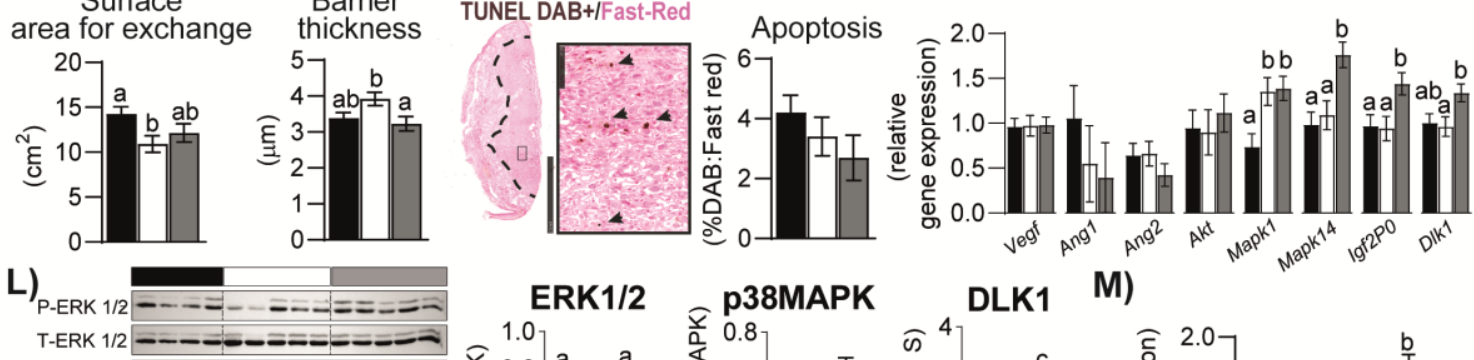

P-P38MAPK

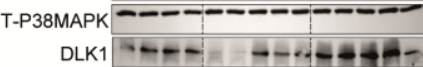
Ponceau S
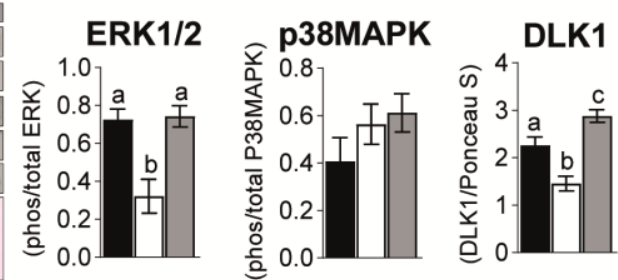

M)

N)

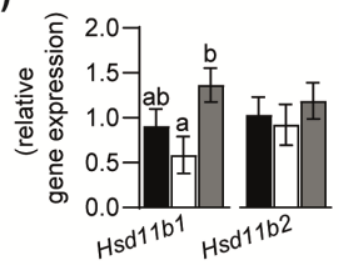

O)

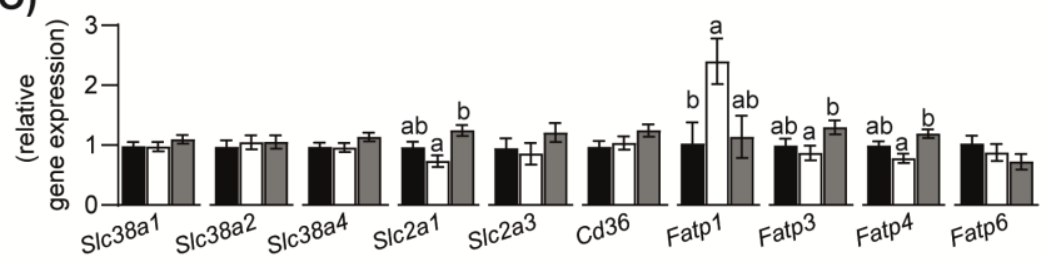

663

Figure 2. Effects of maternal gut microbiome and $B$. breve supplementation during pregnancy on placental structure and placental gene expression on GD16.5. (A) Placenta weight, (B) Placental efficiency determined by dividing fetal by placental mass, (C) Placental regional analysis, (D) Representative staining of placental glycogen with PAS and glycogen abundance, (E) Representative image of lectin and cytokeratin staining for labyrinth zone structural quantification, (F-I) Stereological parameters determined in placental labyrinth zone, (J) Representative image of TUNEL staining for apoptosis quantification in labyrinth zone (KO), Gene expression levels in micro-dissected labyrinth. Data were analyzed by general linear mixed model, taking into account litter size as a covariate and taking each fetus as a repeated measure followed by Tukey multiple comparisons test. ROUT test was used for identification of outlier values. Different superscripts indicate significant differences between groups (a versus b: $P<0.05$; mean $\pm S E M$ ). Placental weight-efficiency was obtained from: SPF (49 fetuses $/ 6$ dams), GF (33 fetuses/5 dams), BIF (34 fetuses/ 6 dams). Laboratorial analysis was performed with: SPF (13-15 placentas/6 dams), GF (10-11 placentas/5 dams) and BIF (14-11 placentas/6 dams). 

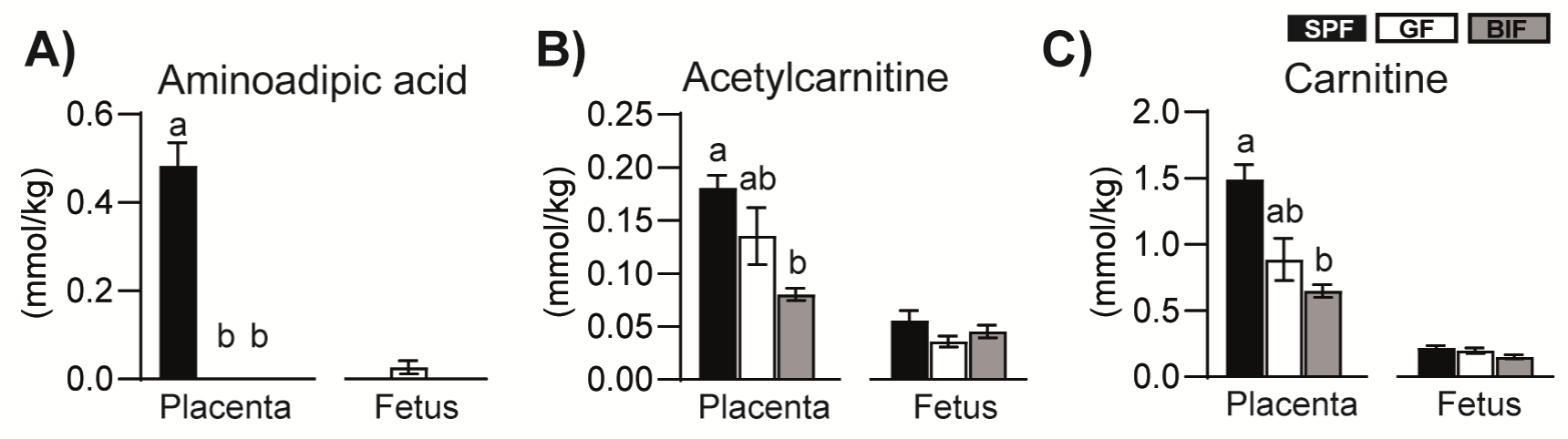

D)

\section{E)}

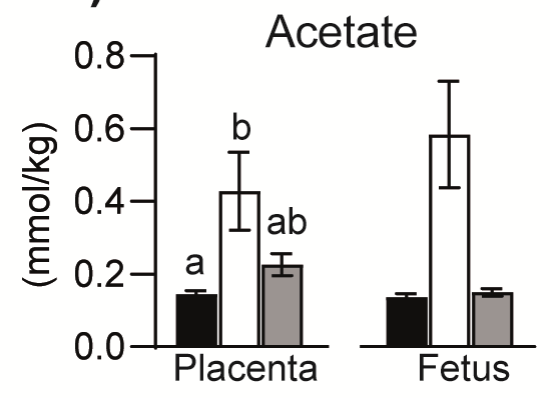

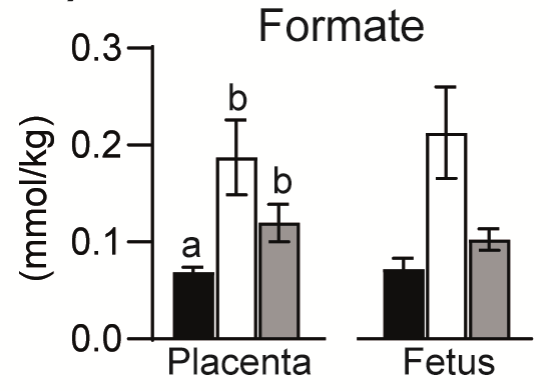

Figure 3. Metabolomic profiling of placental labyrinth zone and fetal liver on GD16.5. Data were analysed by Kruskal-Wallis test followed by multiple comparisons using the Benjamini \& Hochberg false discovery rate method and Dunn's test. ROUT test was used for identification of outlier values. Different superscripts indicate significant differences between groups (a versus $b, P<0.05$ ). Data presented as mean \pm SEM. Number of placentas - livers analysed per group: SPF (8-8/4 dams), GF (6-8/4-5 dams), BIF (6-7/5 dams). 


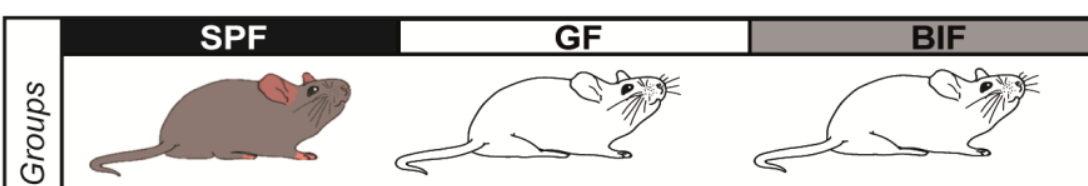

Females C57black6J - day a copulatory plug was found: GD0.5

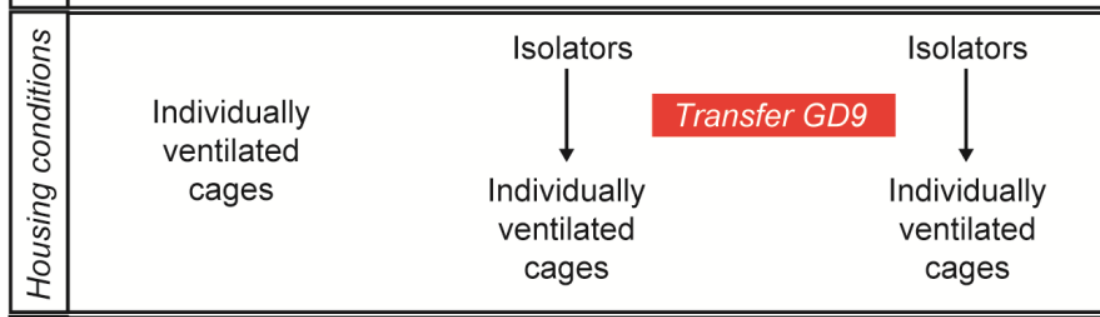

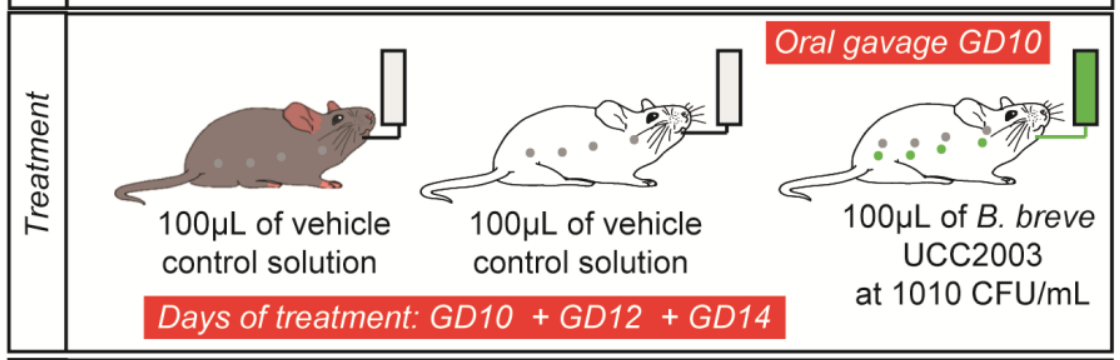

\begin{tabular}{|c|c|c|}
\hline \multirow{6}{*}{ 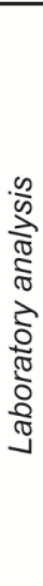 } & \multicolumn{2}{|c|}{ Terminal procedure, days of tissue collection } \\
\hline & GD16.5 & GD18.5 \\
\hline & $\begin{array}{l}\text { Maternal body composition } \\
\text { Maternal metabolism }\end{array}$ & $\begin{array}{c}\text { Fetal liver } \\
\text { RNAseq analysis }\end{array}$ \\
\hline & $\begin{array}{l}\text { Fetal body composition } \\
\text { Fetal glycaemia }\end{array}$ & \\
\hline & $\begin{array}{l}\text { Placental morphology } \\
\text { Placental qPCR analysis } \\
\text { Placental western blotting }\end{array}$ & \\
\hline & $\begin{array}{c}\text { Metabolomics of: } \\
\text { - Maternal caecum content } \\
\text { - Placental labyrinth zone } \\
\text { - Fetal liver }\end{array}$ & \\
\hline
\end{tabular}

689 Supplementary Figure 1. Schematic representation of the experimental design.

690 B. breve was administrated by oral gavage on gestational day (GD) 10, 12 and 14 to germ-free 691 mice. Abbreviations: LZ (placental labyrinth zone); SPF (Specific-Pathogen-Free mouse); GF 692 (Germ-Free mouse); BIF (Germ-Free mouse treated with B. breve UCC2003). 


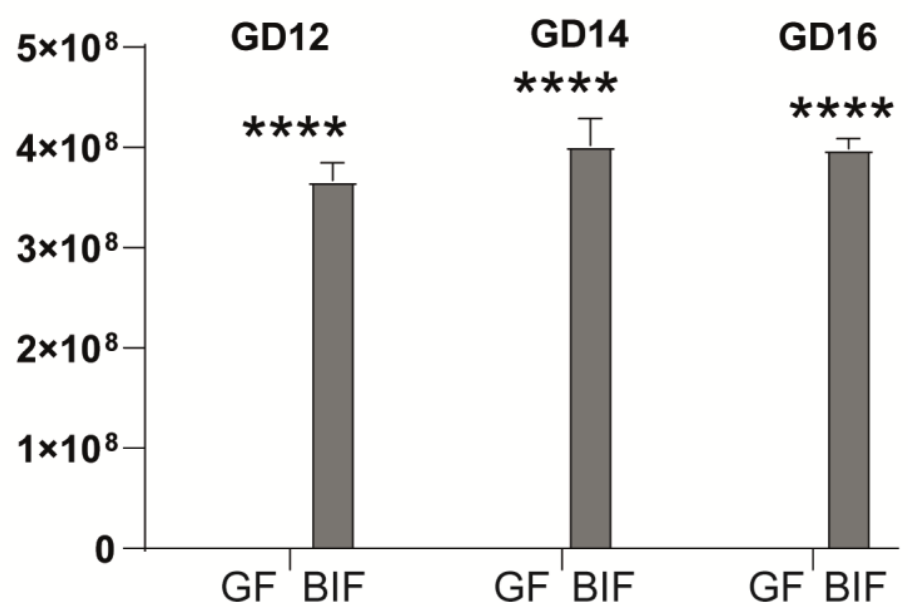
samples on gestational day (GD), 12 and 14. Analysis performed by two-ways ANOVA

\section{Resorption rate}

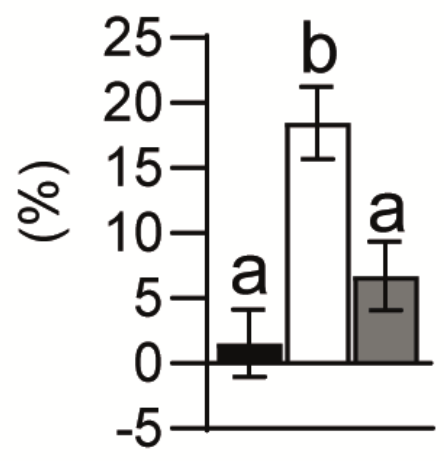

Supplementary Figure 3. Resorption rate defined as the number of resorptions divided by the sum of resorptions and viable fetuses within the litter. Data displayed as mean \pm SEM and analysed by general linear with total litter size as a covariate. Different superscripts indicate significant differences between groups. 


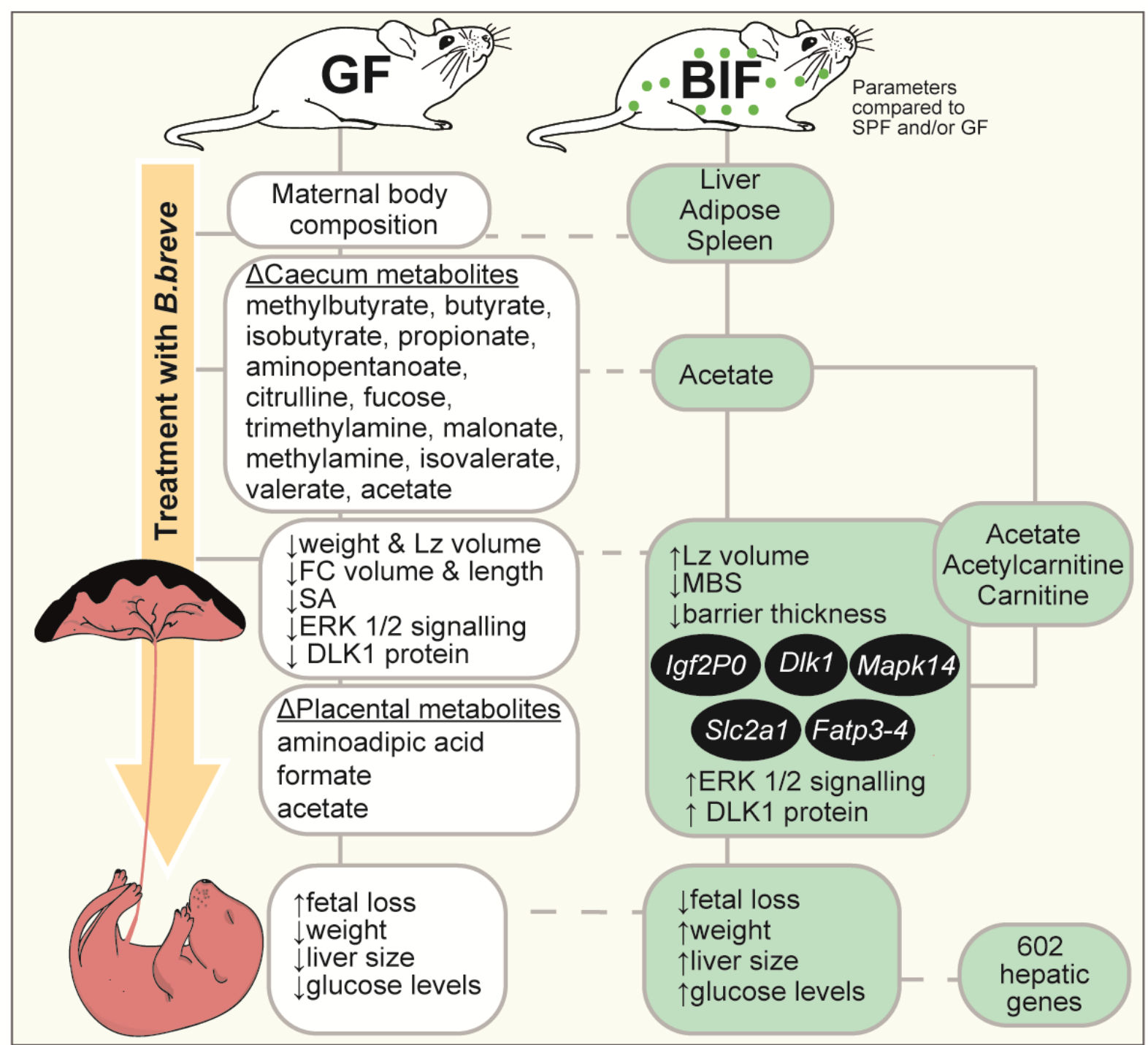

710 Supplementary Figure 4. Summary illustration showing how the maternal gut bacteria 711 and $B$. breve affects mother, placenta and fetus during gestation. Abbreviations: $L Z$ 712 (placental labyrinth zone); SPF (Specific-Pathogen-Free mouse); GF (Germ-Free mouse); BIF 713 (Germ-Free mouse treated with B. breve UCC2003); MBS (Maternal Blood Spaces); FC (Fetal 714 Capillaries); SA (Surface Area). 


\section{Body composition}

\begin{tabular}{|l|l|l|l|l|}
\hline & SPF $(\mathrm{n}=6)$ & GF $(\mathrm{n}=5)$ & $\mathrm{BIF}(\mathrm{n}=6)$ & \\
\hline Hysterectomy weight $(\mathrm{g})$ & $26.01 \pm 0.91$ & $27.87 \pm 0.78$ & $27.17 \pm 0.80$ & NS \\
\hline Digestive Tract (g) & $2.76 \pm 0.03^{\mathrm{a}}$ & $6.83 \pm 0.32^{\mathrm{b}}$ & $7.25 \pm 0.63^{\mathrm{b}}$ & ${ }^{* * *}$ \\
\hline Caecum (g) & $0.66 \pm 0.03^{\mathrm{a}}$ & $3.47 \pm 0.25^{\mathrm{b}}$ & $3.96 \pm 0.41^{\mathrm{b}}$ & ${ }^{* * *}$ \\
\hline Small intestine (g) & $1.66 \pm 0.03^{\mathrm{a}}$ & $2.65 \pm 0.11^{\mathrm{b}}$ & $2.59 \pm 0.14^{\mathrm{b}}$ & ${ }^{* * *}$ \\
\hline Pancreas (mg) & $315.40 \pm 30.12^{\mathrm{a}}$ & $183.40 \pm 24.74^{\mathrm{b}}$ & $190.60 \pm 38.71^{\mathrm{b}}$ & ${ }^{*}$ \\
\hline Gonadal fat (mg) & $433.10 \pm 43.20^{\mathrm{a}}$ & $297.0 \pm 37.02^{\mathrm{ab}}$ & $272.0 \pm 27.35^{\mathrm{b}}$ & ${ }^{*}$ \\
\hline Liver (g) & $2.09 \pm 0.10^{\mathrm{a}}$ & $1.79 \pm 0.05^{\mathrm{ab}}$ & $1.55 \pm 0.08^{\mathrm{b}}$ & ${ }^{* *}$ \\
\hline Spleen (mg) & $117.90 \pm 2.80^{\mathrm{a}}$ & $91.76 \pm 10.60^{\mathrm{ab}}$ & $83.03 \pm 6.72^{\mathrm{b}}$ & ${ }^{*}$ \\
\hline Circulting & & & \\
\hline
\end{tabular}

Circulating metabolites and metabolic hormones

\begin{tabular}{|c|c|c|c|c|}
\hline & SPF $(n=6)$ & GF $(n=5)$ & $\mathrm{BIF}(\mathrm{n}=6)$ & \\
\hline Glucose (mmol/L) & $8.08 \pm 0.78$ & $8.38 \pm 1.18$ & $8.88 \pm 0.74$ & NS \\
\hline Insulin ( $\mu \mathrm{g} / \mathrm{L})$ & $0.12 \pm 0.004$ & $0.19 \pm 0.05$ & $0.20 \pm 0.06$ & NS \\
\hline Leptin $(\mathrm{pg} / \mathrm{mL})$ & $2465 \pm 177.1$ & $2739 \pm 486$ & $2425 \pm 303$ & NS \\
\hline Cholesterol (mmol/L) & $1.33 \pm 0.03$ & $1.56 \pm 0.08$ & $1.41 \pm 0.09$ & NS \\
\hline Triglycerides (mmol/L) & $1.54 \pm 0.08$ & $1.79 \pm 0.14$ & $1.50 \pm 0.11$ & NS \\
\hline Free Fatty Acids ( $\mu \mathrm{mol} / \mathrm{L})$ & $890.6 \pm 101.3$ & $1440 \pm 362$ & $1092 \pm 114.5$ & NS \\
\hline \multicolumn{5}{|l|}{ Caecum Metabolites } \\
\hline & $\operatorname{SPF}(n=3)$ & GF $(n=4)$ & $\mathrm{BIF}(\mathrm{n}=4)$ & \\
\hline 2.Methylbutyrate $(\mathrm{mmol} / \mathrm{Kg})$ & $0.05 \pm 0.01^{a}$ & $0 \pm 0.0^{b}$ & $0 \pm 0.0^{b}$ & * \\
\hline Isobutyrate $(\mathrm{mmol} / \mathrm{Kg})$ & $0.41 \pm 0.24^{a}$ & $0 \pm 0.0^{b}$ & $0 \pm 0.0^{b}$ & * \\
\hline Butyrate $(\mathrm{mmol} / \mathrm{Kg})$ & $12.48 \pm 7.97^{a}$ & $0 \pm 0.0^{b}$ & $0 \pm 0.0^{b}$ & * \\
\hline 5.Aminopentanoate $(\mathrm{mmol} / \mathrm{Kg})$ & $0.33 \pm 0.14^{a}$ & $0 \pm 0.0^{b}$ & $0 \pm 0.0^{b}$ & * \\
\hline Propionate $(\mathrm{mmol} / \mathrm{Kg})$ & $4.48 \pm 1.99^{a}$ & $0 \pm 0.0^{b}$ & $0 \pm 0.0^{b}$ & * \\
\hline Citrulline (mmol/Kg) & $0.30 \pm 0.07^{a}$ & $0 \pm 0.0^{b}$ & $0 \pm 0.0^{b}$ & * \\
\hline Fucose $(\mathrm{mmol} / \mathrm{Kg})$ & $0.08 \pm 0.02^{a}$ & $0 \pm 0.0^{b}$ & $0 \pm 0.0^{b}$ & * \\
\hline Trimethylamine $(\mathrm{mmol} / \mathrm{Kg})$ & $0.06 \pm 0.006^{a}$ & $0 \pm 0.0^{b}$ & $0 \pm 0.0^{b}$ & * \\
\hline Isovalerate $(\mathrm{mmol} / \mathrm{Kg})$ & $0.09 \pm 0.01^{a}$ & $0 \pm 0.0^{b}$ & $0 \pm 0.0^{b}$ & * \\
\hline Methylamine $(\mathrm{mmol} / \mathrm{Kg})$ & $0.05 \pm 0.02^{a}$ & $0 \pm 0.0^{b}$ & $0 \pm 0.0^{b}$ & * \\
\hline Malonate $(\mathrm{mmol} / \mathrm{Kg})$ & $0.09 \pm 0.02^{\mathrm{a}}$ & $0 \pm 0.0^{b}$ & $0 \pm 0.0^{b}$ & * \\
\hline Valerate $(\mathrm{mmol} / \mathrm{Kg})$ & $0.57 \pm 0.27^{a}$ & $0 \pm 0.0^{b}$ & $0 \pm 0.0^{b}$ & * \\
\hline Acetate $(\mathrm{mmol} / \mathrm{Kg})$ & $35.49 \pm 20.66^{a}$ & $0.55 \pm 0.06^{b}$ & $3.79 \pm 1.64^{a b}$ & * \\
\hline
\end{tabular}

Table 1. Effects of maternal gut microbiome and $B$. breve administration during pregnancy on maternal body composition, circulating metabolites and hormones in maternal serum, and metabolites in caecum. Body composition and metabolites/hormones in serum were analyzed by one-way ANOVA followed by Tukey multiple comparisons test. Metabolites in maternal caecum were analysed by Kruskal-Wallis test followed by multiple comparisons using the Benjamini \& Hochberg false discovery rate method and Dunn's test. ROUT test was used for identification of outlier values. Different superscripts indicate significant differences between groups (a versus b, $\mathrm{P}<0.05)\left({ }^{*} \mathrm{P}<0.05 ;{ }^{* *} \mathrm{P}<0.01 ;{ }^{* * *} \mathrm{P}<0.001\right.$; 
$743{ }^{* * * *} \mathrm{P}<0.0001$; NS: not significant). Data presented as mean \pm SEM. The number of dams used 744 for each group is annotated on the table.

745 Supplementary Table 1. List of differentially expressed genes and pathways detected in 746 the liver RNA-Seq on day 18.5 of gestation.

747

748 Supplementary Table 2. List of metabolites analysed in maternal caecum, placental 749 labyrinth zone and fetal liver on day 16.5 of gestation. 
751

\begin{tabular}{|c|c|c|}
\hline & Forward & Reverse \\
\hline Hprt & CAGGCCAGACTTTGTTGGAT & TTGCGCTCATCTTAGGCTTT \\
\hline Ubc & GGAGTCGCCCGAGGTCA & AAAGATCTGCATCGTCTCTCTCAC \\
\hline Vegf & GAAGCTACTGCCGTCCGATT & CTTCATCGTTACAGCAGCC \\
\hline Ang1 & GAAGCAACTTCTCAACAGACA & TTCTTTGTGTTTTCCCTCCATT \\
\hline Ang2 & CTTCTACCTCGCTGGTGAAGAG & GCTAAAATCACTTCCTGGTTGG \\
\hline Akt & GCCGCCTGATCAAGTTCTCC & TTCAGATGATCCATGCGGGG \\
\hline Mapk1 & TGCTTTCTCTCCCGCACAAA & GGCCAGAGCCTGTTCAACTT \\
\hline Mapk14 & AGCTGTCGAGACCGTTTCAG & GATGGGTCACCAGGTACACG \\
\hline Dlk1 & GAAAGGACTGCCAGCACAAG & CACAGAAGTTGCCTGAGAAGC \\
\hline $\operatorname{lgf} 2 P 0$ & GAGGAAGCTCTGCTGTTTGG & CAAAGAGATGAGAAGCACCAAC \\
\hline Hsd11b1 & GAGGAAGGTCTCCAGAAGGTA & ATGTCCAGTCCGCCCAT \\
\hline Hsd11b2 & GGCTGGATCGCGTTGTC & CGTGAAGCCCATGGCAT \\
\hline S/c38a1 & CGGCGCCTTTCCCTTTATTTC & CCGTTAACTCGAGGCCACTT \\
\hline Slc38a2 & TTCTGATTGTGGTGATTTGCAAGAA & CAGGATGGGCACAGCATACA \\
\hline Slc38a4 & AAGGTAGAGGCGGGAAAGGG & AGGAACTTCTGACTTTCGGCA \\
\hline Slc2a1 & GCTTATGGGCTTCTCCAAACT & GGTGACACCTCTCCCACATAC \\
\hline Slc2a3 & GA TCGGCTCTTTCCAGTTTG & CAA TCA TGCCACCAACAGAG \\
\hline Cd36 & ATGGGCTGTGATCGGAACTG & GTCTTCCCAATAAGCATGTCTCC \\
\hline Fatp1 & GGCTCCTGGAGCAGGAACA & ACGGAAGTCCCAGAAACCAA \\
\hline Fatp3 & GAGAACTTGCCACCGTATGC & GGCCCCTATATCTTGGTCCA \\
\hline Fatp4 & GATTCTCCCTGTTGCTCCTGT & CCATTGAAGCAAACAGCAGG \\
\hline Fatp6 & AACCAAGTGGTGACATCTCTGC & TCCATAAAGTAAAGCGGGTCAG \\
\hline Kras & ACAGGCTCAGGAGTTAGCAAG & ACACAGCCAGGAGTCTTTTCT \\
\hline Nras & CAAAGCAAGCCCACGAACTG & CATCGTCACTGCTGTTGAGC \\
\hline
\end{tabular}

752 Supplementary Table 3. List of primers used for placental labyrinth zone qPCR 